\title{
The "Highly Versatile Single Port System” for laparoscopic surgery: Introduction and first clinical application
}

\author{
S. Can ${ }^{1}$, H. Mayer ${ }^{3}$, A. Fiolka ${ }^{1}$, A. Schneider ${ }^{1}$, D. Wilhelm ${ }^{1,2}$, H. Feussner ${ }^{1,2}$, A. Knoll ${ }^{3}$ \\ ${ }^{1}$ Research Group MITI, Klinikum rechts der Isar, Technische Universität München, Germany \\ ${ }^{2}$ Department of surgery, Klinikum rechts der Isar, Technische Universität München, Germany \\ ${ }^{3}$ Chair of Robotics and Embedded Systems, Technische Universität München, Germany
}

\begin{abstract}
In minimally invasive surgery, trauma to the patient can be reduced by diminishing the number of entry ports. In addition a single port access, attaining "high flexibility" in laparoscopic interventions is also of utmost importance. We developed the "Highly Versatile Single Port System" (HVSPS), a two-armed device with an additional semi-flexible telescope, to meet these requirements. Both manipulators and the telescope are inserted independently through a three-channel trocar which is guided by a four degree of freedom telemanipulator. Furthermore, a simulation of the complete system including the surgical environment is programmed for user training and design optimization. The first gallbladder could be successfully resected in an animal experiment using the HVSPS. The manipulators were introduced into the abdominal cavity through a single incision. After the ligation of the cystic duct and artery with coagulation current, the gallbladder was held with a grasper and dissected using a TT knife. The complete surgical intervention, without technical set-up, was accomplished within 110 minutes. For the first time, the feasibility of laparoscopic cholecystectomy using the HVSPS has been shown in this study. However, such elaborate systems bring up new challenges such as the definition of an adequate human-machine interface and new control approaches for retroflective manipulations which are possible with the kinematic structure of the HVSPS.
\end{abstract}

Keywords- single port access, flexible instrument, laparoscopic telemanipulator, laparoscopic surgery, minimally invasive surgery

\section{INTRODUCTION}

The tendency towards further trauma reduction can be realized by reducing the number of ports for laparoscopic interventions ("single port surgery"). Accordingly, the need for a multifunctional (viewing and manipulation) single port operation system is growing [1]. Beyond of the single port access, "high flexibility" is a substantial requirement for laparoscopic surgery which is investigated by several research institutes [2-4]. The intra-abdominal flexibility of stiff laparoscopic instruments limited due to the entry port is augmented with additional distal articulations.
An additional stimulus is given by the introduction of socalled "Natural Orifice Transluminal Endoscopic Surgery" (NOTES), since this type of surgery depends even more upon a suitable single lumen universal tool. [5] The first approach devoted to this problem is the endoscope-based Octopus system as developed by Swanstrom et al [6]. Other systems developed for single port surgery or NOTES interventions are presented in $[7,8]$. However, these systems have a small working range, limited flexibility and opposite or retroflective working is not possible. Thus a new mechatronic support platform is required to increased penetration of minor access surgery and NOTES.

We developed the "Highly Versatile Single Port System" (HVSPS) to overcome these drawbacks. The idea is, literally spoken, to bring the surgeon's head, shoulders and arms into the abdominal cavity to regain the same flexibility as in open surgery. Compared to other systems, only a single four degrees of freedom telemanipulator is required to guide the HVSPS. The surgeon controls the manipulators in an intuitive manner through a master console. Our aim was to prove the maturity for an in vivo surgical operation.

\section{Material \& Methods}

\section{A. The HVSPS mechatronic support platform}

Using the know-how and partially the hardware of flexible endoscopes we designed a two armed device with two manipulators and a semi-flexible telescope. Fig. 1 shows a schematic design of the complete HVSPS. Both manipulators and the telescope are inserted independently through an insert with three lumens. This ensemble is introduced gas-tightly into the abdominal cavity using a 33 $\mathrm{mm}$ trocar and guided over a telemanipulator attached to the insert. The guiding manipulator with four degrees of freedom allows the pivoting of the system at the fulcrum, a linear movement into the body and the rotation of the complete HVSPS.

The hollow manipulators with five degrees of freedom have an outer diameter of $12 \mathrm{~mm}$. Flexible instruments are introduced through the central channels and they are 
controlled and changed manually. The bendable section of the manipulators with two degrees of freedom, resembling to the human wrist, has a length of $75 \mathrm{~mm}$ followed by a 50 mm length hollow tube and an elbow articulation with one degree of freedom. Two additional degrees of freedom at the distal end of the manipulators provide a rotation of 270 degrees in each direction and a linear movement of $80 \mathrm{~mm}$ into the abdominal cavity. Visualization of the situs is realized by a flexible telescope with $10 \mathrm{~mm}$ diameter and five degrees of freedom. This solution was implemented by a commercial endoscope with $6 \mathrm{~mm}$ diameter, which is inserted through a $10 \mathrm{~mm}$ tube with a distal deflection of 30 degrees. Given this ensemble, the telescope can be moved in an S-form so that the instruments of both manipulators can be observed in their entire working range.

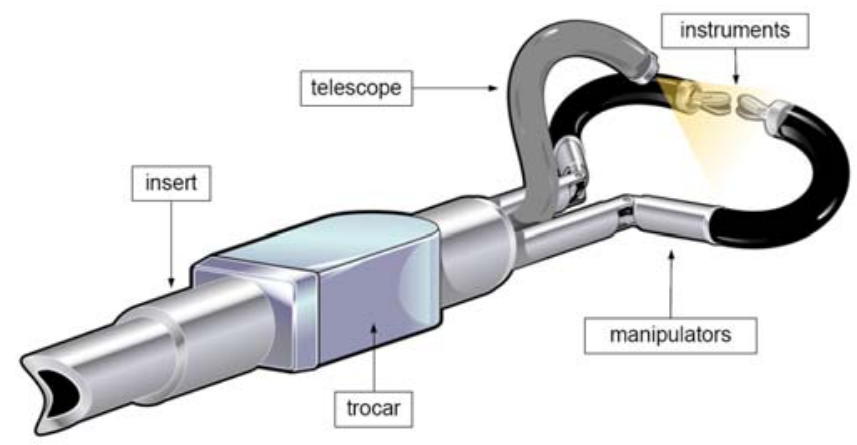

Fig. 1 Schematically drawing of the "Highly Versatile Single Port System”

Both manipulators are partially automated and controlled over a real-time Matlab-Simulik application. In its current state (two distal articulations of each manipulator), a total of six degrees of freedom is motorized and steered using bowden wires. The drive system is placed to the periphery, two meter away from the patient. The actual state of the development and control is demanding so that an interdisciplinary training was indispensable for reasonable interventions. Accordingly, the complete system had to be operated by a team composed of several physician and engineers. One surgeon controls the manipulators over two joysticks, a second one controls manually the flexible instruments guided through the manipulators, a gastroenterologist guides and controls manually the semiflexible telescope and an engineer operates the nonmotorized degrees of freedom of the system.

In a first experiment, the complete system was tested on the ex-vivo ELITE trainer, a human "mock-up" for teaching laparoscopic and NOTES procedures [9]. After this evaluation the first laparoscopic in-vivo cholecystectomy was performed in an animal study.

\section{B. Simulation environment of the platform}

The simulation of elaborate mechatronic systems plays a central role regarding the prediction of development features, system functionality and teaching facilities. We started developing a simulation of the complete system parallel to the hardware design. Below, some of the important reasons for the programmed HVSPS simulation environment are listed.

- Design optimization of the mechanical prototype

- Development of new surgical instruments

- Identification of the appropriate human-machine interface

- User training for the physicians

- Parallel usability on multiple workstations

A complete surgical scenario with the HVSPS attached to the SoloAssist (Aktormed, Barbing, Germany) telemanipulator and mounted on an operating table could be implemented in the Coin3D open source graphics development tool (Fig. 2). All the functionalities are currently controlled using a single input device. It is decisive using a simulation to predict the feasibility of a mechatronic design and identify thereby difficulties and limitations of the concept at early stages. Thus, questions like "Is retroflective manipulation feasible under direct camera view?" could be answered. Furthermore the kinematic structure and dimensions, e.g. the flexibility of the telescope like a "gooseneck", could be defined using a simulation of the system.

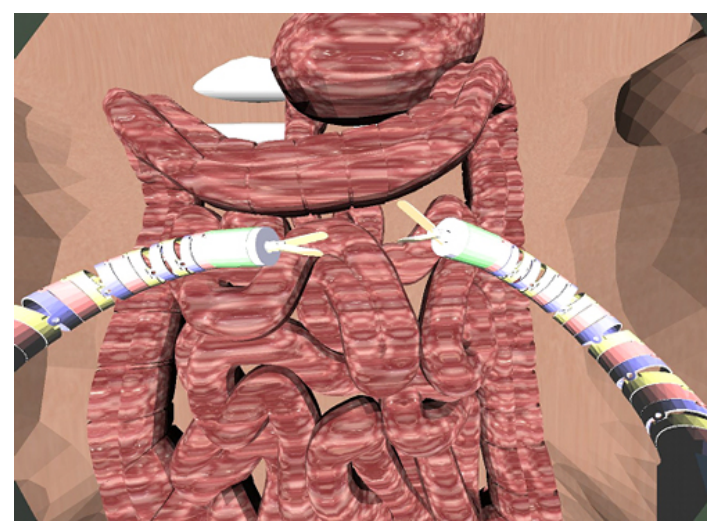

Fig. 2 Simulation environment of the HVSPS (view of the flexible telescope)

Instruments with multiple articulation and high flexibility, e.g. "snakelike" structures, lead to an overdetermined kinematics which is difficult to handle and requires new human-machine interface approaches. The simulation provides a platform to evaluate different input 
devices to determine an adequate interface for the HVSPS. Since it is clear that one surgeon cannot control and handle the complete system in its actual state, the surgical intervention scenario could be animated on the simulation to assign the different tasks of the operation (For example a surgeon controls both manipulators and commands the operation workflow and a gastroenterologist guides the telescope).

It is very practical and effective for surgeons to be trained on a simulation for new surgical systems such as the HVSPS before applying it under real circumstances. The surgeon can get familiar with the system and all-important the interdisciplinary working with several physicians can be trained as well. The physicians were preliminary introduced to the HVSPS using simple input devices. The simulation environment for surgical training provides additional advantages such as ease of use on arbitrary amount of workstations, low running costs and no mechanical wear out.

Furthermore the simulation can support the remote surgeon during real application on the patient by representing the instruments even when they are occluded in reality by other objects.

\section{Future extensions of the platform}

The integration of the HVSPS system into the ARAMIS platform is planned for the near future. This platform comprises a telemanipulator and a master console to guide minimally invasive instruments [10]. The console consists of two Sensable PHANTOMs utilized as input as well as force feedback devices. In addition, it provides a 3D viewer to display the images of a stereo endoscope. This master console could be ideally used to steer the HVSPS manipulators by Cartesian control.

The ARAMIS hardware is already mapped to a comprehensive simulation environment providing 3D models of all devices and an interactive user interface. In addition, a key-framing interface, as known from computer animations, can be used for operation planning by users with limited background regarding robotics and low-level control. This simulation environment has already been used to perform automated knot-tying in a minimally invasive scenario. The developed techniques are independent of a specific scenario, and therefore, can easily be transferred to a "Single-Port" application like a HVSPS-based cholecystectomy. The ARAMIS simulation environment additionally provides beneficial features like sophisticated collision detection and a multi-tier software platform for robot control.

\section{RESULTS}

\section{A. Experimental ex-vivo evaluation on the ELITE trainer}

Several evaluations of the HVSPS on the ELTE trainer were performed for this study. Beyond teaching and user training, functionality tests, coordination and handling of the system were the main purpose of these evaluations. In first trials the system was deployed for manipulating human mock-up intestine. (Fig. 3) During these tests the performed manipulations could be followed by direct view on the system. With grasping, cutting and ligation tasks the surgeons could get familiar to the system and extract the assignment of tasks for further application.

After several experiments we accomplished the first gallbladder dissection on the ex-vivo ELITE trainer under circumstances close to the real in-vivo cholecystectomy. More than two hours elapsed for accomplishment of the gallbladder dissection. This interdisciplinary training was indispensable for the intended in-vivo cholecystectomy in a swine experiment.

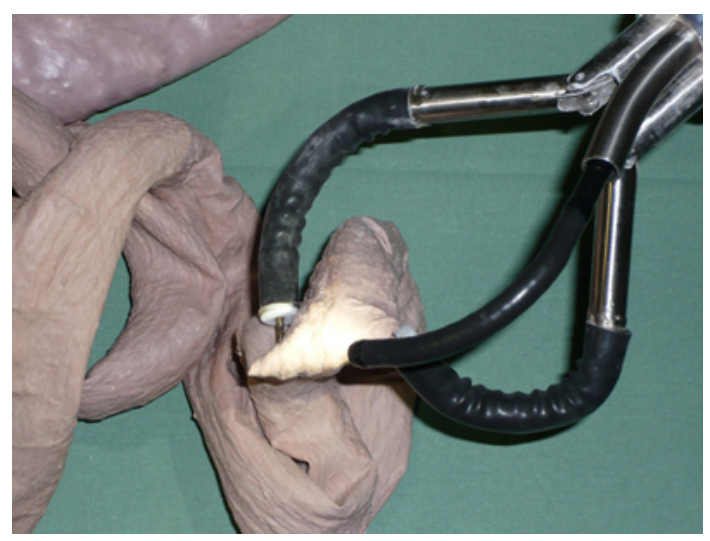

Fig. 3 Evaluation of the HVSPS on a human mock-up intestine

\section{B. Surgical in-vivo evaluation; accomplishment of the first cholecystectomy in an animal experiment}

The first gallbladder was successfully resected with the HVSPS in an animal experiment under general anesthesia. The complete surgical intervention, without technical setup, was accomplished in 110 minutes. After insufflation of the peritoneum with a Verres needle, the HVSPS was introduced into the abdominal cavity through one incision in the middle of the abdomen. The manipulators were in a straight position during the introduction and expanded afterwards in the peritoneal cavity. With a retractor introduced through an auxiliary incision, the liver could be retained out of the operating field. 
The physician controlling the flexible endoscope observed the situs after introduction and helped guiding the manipulators to face the gallbladder. Two flexible instruments (grasper, scissors, etc.) were introduced afterwards through the manipulators, which could be exchanged within seconds for different tasks.

After the ligation of the cystic duct and cystic artery with coagulation current, dissection of the gallbladder was achieved by using grasping and cutting instruments. Fig. 4 shows how the gallbladder is hold with a grasper through the left manipulator and dissected by using a TT knife introduced through the right one. The opposition of the manipulators was essential for intuitive working. The gallbladder was recovered through the main incision after a last check up for bleeding and complication. The manipulators were steered to a straight position so that the complete system could be pull out of the abdomen and finally both incisions were sutured.

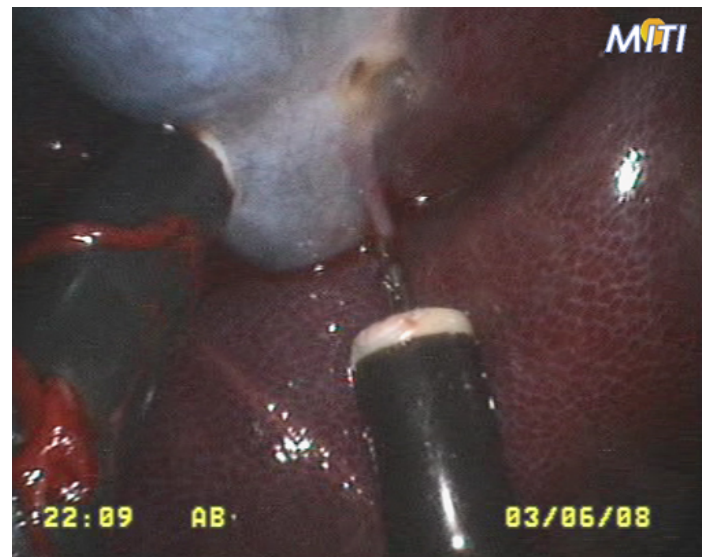

Fig. 4 First cholecystectomy with the HVSPS in an animal experiment

The complete surgical intervention was managed by commands of the surgeon who controlled the manipulators. Coordination of three physicians was essential for the performance and quality of the intervention. Though, the communication during the operation and occasional disaccord of the physicians was time-consuming.

\section{CONCLUSION}

Laparoscopic cholecystectomy using the HVSP support system is feasible. However, compared to the conventional laparoscopic cholecystectomy, the operation took considerably longer. This is resulting from the still very difficult control of the various degrees of freedom of the system and the essential coordination of the individual actions of the team members. This extended time can be reduced by optimizing the fully automated HVSPS, introducing an intuitive human-machine interface and an integrated simulation and planning environment like the one of the ARAMIS system.

It is also possible to use the HVSPS for retroflective interventions which were not possible yet. However, a new control design should be developed, since it was impossible to work in a head over position. Further work will be focused on the intuitive human-machine interface and the integrated intelligence required for such elaborate systems.

\section{ACKNOWLEDGMENT}

We gratefully thank Karl Storz GmbH in Tuttlingen for their support and providing the mechanical hardware. This project was supported in part by the Bavarian Research Foundation (BFS).

\section{REFERENCES}

1. Feussner H, Can S, Fiolka A, Schneider A. Hybrid surgery-the way towards notes the challenge for computer science. Biomedical Imaging: From Nano to Macro, 2008 ISBI 2008 5th IEEE International Symposium on 2008;1383-6.

2. Ikuta K, Sasaki K, Yamamoto K, Shimada T. Remote Microsurgery System for Deep and Narrow Space-Development of New Surgical Procedure and Micro-robotic Tool, Proc. of 5th International Conference on Medical Image Computing and Computer-Assisted Intervention(2002) 163-172

3. Song H, Chung J, Kim K, Lee J. The Development of human-arm like manipulator for Laparoscopic Surgery with Force sensing, Industrial Technology, 2006. ICIT 2006. IEEE International Conference on (2006) 1258-1262.

4. Yamashita H, Iimura A, Aoki E, Suzuki T, Nakazawa T, Kobayashi $\mathrm{E}$, et al. Development of endoscopic forceps manipulator using multislider linkage mechanisms. Journal of Japan Society of Computer Aided Surgery 2005;7(2):201-4.

5. Rattner D, Kalloo A. ASGE/SAGES Working Group on Natural Orifice Translumenal Endoscopic Surgery. Surgical Endoscopy 2006;20(2):329-33.

6. Swanstrom LL, Kozarek R, Pasricha PJ, Gross S, Birkett D, Park PO, Saadat V, Ewers R, Swain P. Development of a new access device for transgastric surgery, Journal of Gastrointestinal Surgery 9 (2005) 1129-1137.

7. Raman JD, Bensalah K, Bagrodia A, Stern JM, Cadeddu JA. Laboratory and Clinical Development of Single Keyhole Umbilical Nephrectomy. Urology 2007;70(6):1039-42.

8. Thompson CC, Ryou M, Rothstein RI, Fong DG, Pai RD, Smith B.S. P,. Weitzner B.S. BD (May 21 2007). Stomach - Direct Drive Endoscopic System for Endoluminal and NOTES Applications. The DAVE Project. (http://daveproject.org/viewfilms.cfm?film_id=612)

9. Gillen S, Doundoulakis E, Schneider A, Fiolka A, von Delius S, Meining A, Friess H, Feussner H. The "ELITE" model: Construct validation of a new training system for Natural Orifice Translumenal Endoscopic Surgery (NOTES), Endoscopy (2008) "submitted”

10. Mayer H, Nagy I, Knoll A, Braun EU, Bauernschmitt R. Human Computer Interfaces of a System for Robotic Heart Surgery. Proceedings of the Second IASTED International Conference on Human-Computer Interaction, 31-36. 2007. Chamonix, France. 